\title{
Diagnostische Notizen
}

Die Glaukome nach Operationen des grauen Stars und des Nachstars.

Dr. Stölting-Hannover berichtethierüber in einer umfassenden Mitteilung. die besonders lehrreich wird durch mitgeteilte eigene Erfahrungen,

Folgende Gruppen sind nach dem Verf. zu unterscheiden:

Glaukom, kommt vor:

Infolge von Einklemmung der Linsenkapsel in die Wunde a) unter demBilde der serösen Iritis; b) unter dem Bilde chron. inflammatorischen Glaukoms.

Infolge Einwanderung des Epithels durch die Hornhaut $\lambda$ vunde auf die Iris.

Nach Diszission.

Für jede Gruppe werden Belegfälle mitgeteilt, außerdem die Literatur kritisch gesichtet.

Der zur Gruppe I besprochene Fall ist besonders interessant und genauen Studiums für jeden Praktiker $\Lambda$ vert. Das betreffende Auge kam trotz glatten Extraktions- und Heilungsverlaufes (v. Graefes Modifizierte Linearextraktion mit Konjunktivallappen) 3 Monate nach der Operation zur Enukleation.

Die Erklärung des Zustandekommens des glaukomatösen Vorgangs auf Grund der klinischen Beobachtung und des anatomischen Befundes lautet:

,,Die Kapseleinklemmung in die Hornhautwunde drückte den Irisstumpf an die hintere Hornhautwand und schloß einen Teil der

$\mathrm{Z}^{1} / 8$ ítschrít für Augenheilkunde. Bd. XXVIII. Heft 1.

6

82

Diagnostisch $\theta$ Notizen.

Abfluß $\lambda$ vege der Vorderkammer. Gleichzeitig wirkte die Zerrung an der Zonula als ein Moment, das die Sekretion von Kammerwasser mächtig anregte. Solange die Wunde filtrierte, traten Unregelmäßig-keiten nicht hervor, sobald aber der Verschluß der Extraktionswunde, hier nach 30 Tagen, perfekt war, kam es zur ersten Drucksteigerung. Sie ließ sich noch durch Eserin und die üblichen antiglaukomatösen Maßnahmen beseitigen, erschien aber wieder und wieder. Das unter diesen besonderen Umständen abgesonderte Kammerwasser war nicht mehr normal, sondern je länger der Prozeß dauerte, um so eiweißreicher wurde es. Mehr und mehr kam es zum Verschluß der noch vorhandenen Abflußwege. Das Glaukom nahm nun einen schweren Charakter an. (Beschläge an der Hornhaut traten auf, weil das Kammerwasser mit fremdartigen Stoffen beladen war, daher Vérdacht auf Cyclitis, der aber unberechtigt war.) Aehnliche Fälle beschrieben Treacher - Collins, Landsberg, Pagen-stecher, Dalèn. Die Abweichungen $\lambda$ verden besprochen.

DieGruppell umfaßt die Fälle, , in welchen Epitheleinwanderungen in die Vorderkammer stattfanden; sie haben mit der Extraktion der Linse direkt nichts zu tun. Hier ist nur die Eröffnung der Bulbuskapsel und das Offenbleiben der Wunde von Bedeutung. Das Epithel kriecht durch die offene Hornhautwunde auf die Iris hinüber und füllt als Schlauch den Wundkanal. Nachträglicher peripherer Wundverschluß läßt eine Cyste der vorderen Kammer entstehen, die durch stete Ver-größerung alle Exkretionswege blockiert”. 
Die bekannten Fälle anderer Autoren werden besprochen, ein eigener genau beschriebener Fall, der zur anatomischen Untersuchung kam, $\Lambda$ vird angefügt und an ihm die Schwierigkeit der Diagnose und Prognose in praxi demonstriert.

Die Gruppe III: Glaukom nach Diskussion im Anschluß an Kata-raktextraktion $\Lambda$ vird nur kurz besprochen, doch auch mit einem eigenen Fall belegt (dem einzigen des Verfassers). Als bedeutsam wird bezeichnet, , daß Drucksteigerung weit häufiger bei Diszissionen beobachtet wird, $\Lambda$ velche der einfachen Extraktion folgen, als bei solchen nach der kom-binierten Methode. Die Autoren, u. A. v. Graefe und Bowman sind darüber einig, daß Läsionen der Sphinkterpartie bei enger Pupille die Ursache dazu abgäben".

Unter den eigentlichen Gründen, den die Drucksteigerung be-fördernden Momenten, die sich nach Gruppen nicht gut scheiden lassen, spielt eine große Rolle der verzögerte Wundschluß, ferner Anzeichen im Wundverlauf.

Für die Drucksteigerung nach Diszission von Nachstaren glaubt v. Graefe zwei Gründe anschuldigen zu sollen, zunächst Aufquellung von Linsenmassen, die im Kapselsack eingeschlossen waren und durcli die Operation frei wurden, sodann aber Zerrung an den Ciliarfirsten durch Dislokation des Kapselsackes1).

1.) Daher die Vorschriften v. Graefes : Ni $\beta$ bei enger, den Mydriatiois widerstehender Pupille eine Kapseldiszission vornehmen, sondern vorher Iridektomie anlegen. - Nie den Pupillarrand bei der Diszission berühren

Therapeutische Umschau.

S3

Zuzugeben ist, daß die Glaukome nach Diszission eine Sonder-stellung einnehmen; sie sind im allgemeinen sehr gutartig (wenn auch nicht immer) und heilbar.

Die Prognose der Glaukome nach Staroperationen im allgemeinen hat Dalèn in einer Statistik zu finden gesucht. Man kann sie noch nicht als hinreichend abgeschlossen ansehen, da bei vielen Fallen Besserung noch erwartet wird.

Die Angaben sprechen aber dafür, daß die einfache Extraktion weniger oft zum Glaukom führt als die kombinierte.

,,Das Wichtigste ist aber, daß das Glaukom, das nach der einfachen Methode entstanden ist, sei es nach primärer Extraktion, sei es nach Diszission, gutartig auftritt, und daß bei der kombinierten Methode das Gegenteil der Fall ist1).

Die Nutzanwendung, die man aus alien Erfahrungen machen muß, scheint dem Verf. hauptsächlich darin zu bestehen, daß man die Ge-fahren der Iris- und $\mathrm{Ka}<$ pseleinklemmung vermeiden soil.

Die Lösung des Problems ist aber in der Praxis nicht sowohl Ver-meidung der Iridektomie, sondern die Extraktion mit nachfolgender kleiner peripherer Iridektomie. (Nach Pßüger-Heß.) Hierdurch werden die Gefahren auf ein Minimum reduziert. Freilich ist zu sagen: Nicht sämtliche Katarakte können so operiert werden. (Vorfall der Iris vor das Messer, gelegentliche Unmöglichkeit, die Linse ohne Iridektomie zu entbinden.)

Alle Maßnahmen werden erleichtert durch Anwendung der Skopo-lamin-Morphium-Narkose, die Verf. in alien geeigneten Fallen in neuerer Zeit verwandte und für eine wertvolle Errungenschaft auch für die Augenheilkunde halt. (v. Cfraefes Arch. f. Ophthalm. 1912. H. 3.) Junius.

Therapeutische Umschau.

Über die Cyklodialyse. Von Dr. W. Meisner und Priv.-Doz. Dr. C. H.

Battler. Arch. f. Augenheik. 1912. 71. Heft 1 und 2. 
Bezüglich der Beurteilung des Erfolges von 54 in Königsberg operierten Glaukomfällen stellen die Autoren mit Recht sehr strenge Kriterien auf. Nur solche Fälle können nach ihnen als Erfolge be-trachtet $\Lambda$ verden, bei denen während eines viertel bis eines halben Jahres weder der Visus sinkt, noch das Gesichtsfeld eine weitere Ein-«chränkung erfährt, und der Druck auch ohne Wirkung eines Miotikums mit dem Schiötzschen Tonometer sich als normal erweist. Von 39 Fallen,

L. Linsenkapsel leicht und geräumig aufschlitzen. - Nie die opaken Teile der Kapsel angreifen, nur die durchscheinenden, für die Nadel nachgiebigen Partien zerteilen. 